\title{
Case report of an epidural cervical Onchocerca lupi infection in a 13-year-old boy
}

\author{
Tsinsue Chen, MD, ${ }^{1}$ Karam Moon, MD, ${ }^{1}$ Daphne E. deMello, MD, ${ }^{2}$ Iman Feiz-Erfan, MD, ${ }^{4,5}$ \\ Nicholas Theodore, MD, ${ }^{1}$ and Ratan D. Bhardwaj, MD, PhD ${ }^{3}$
}

\begin{abstract}
1Department of Neurological Surgery, Barrow Neurological Institute, St. Joseph's Hospital and Medical Center; ${ }^{2}$ Department of Pathology and Laboratory Medicine, Phoenix Children's Hospital; ${ }^{3}$ Division of Neurological Surgery, Barrow Neurological Institute, Phoenix Children's Hospital; ${ }^{4}$ The University of Arizona, College of Medicine-Phoenix; and ${ }^{5}$ Division of Neurosurgery, Maricopa Medical Center, Phoenix, Arizona
\end{abstract}

\begin{abstract}
A 13-year-old boy presented with fever and neck pain and stiffness, which was initially misdiagnosed as culture-negative meningitis. Magnetic resonance images of the brain and cervical spine demonstrated what appeared to be an intradural extramedullary mass at the C1-3 level, resulting in moderate cord compression, and a Chiari Type I malformation. The patient underwent a suboccipital craniectomy and a C1-3 laminectomy with intradural exploration for excisional biopsy and resection. The lesion containing the parasite was extradural, extending laterally through the C2-3 foramina. Inflammatory tissue secondary to Onchocerca lupi infection was identified, and treatment with steroids and doxycycline was initiated. At the 6-month follow-up, the patient remained asymptomatic, with MR images demonstrating a significant reduction in lesional size. However, 10 weeks postoperatively, the infection recurred, necessitating a second operation. The patient was treated with an additional course of doxycycline and is currently maintained on ivermectin therapy. This is the second reported case of cervical 0 . lupi infection in a human. In the authors' experience, oral doxycycline alone was insufficient in controlling the disease, and the addition of ivermectin therapy was necessary.
\end{abstract}

http://thejns.org/doi/abs/10.3171/2014.12.PEDS14462

KEY WORDS cervical infection; Onchocerca lupi; parasite; spinal infection; spine

$\mathrm{S}$ PINAL zoonotic parasitic infections are uncommon, with neurocysticercosis (Taenia solium) via swine and echinococcosis (Echinococcus granulosus) via canines being the most common CNS parasitic infections known to infect the human spine. Approximately 1\%-3\% of patients with intracranial neurocysticercosis infections also have spinal involvement, with evidence of either a mass lesion or a more diffuse arachnoiditis. While isolated spinal cysticercosis without intracranial involvement is rare, patients with spinal echinococcosis present more often with primary spinal infection. ${ }^{1,3,5,7-14,17-20,22}$ In a recent review of 467 cases of spinal echinococcosis by Neumayr et al. ${ }^{13} 17.9 \%$ were primary spinal infections without intracranial involvement.

We describe a cervical epidural infection caused by the nematode species Onchocerca lupi. Onchocerca is a ge- nus of nematodes with 34 different species, which primarily parasitize nonhuman hosts. ${ }^{9}$ The only well-established species known to cause human infection is $O$. volvulus, which causes onchocerciasis or "river blindness," primarily in Africa. ${ }^{2} O$. lupi is the only other species that has been reported to infect humans ( 7 cases total). Here we present the second reported case of $O$. lupi infection in the epidural cervical spine. The remaining 5 reported cases of O. lupi manifested as ocular onchocercosis. ${ }^{5}$

\section{Case Report}

History and Physical Examination

A 13-year-old Native American boy residing in northeastern Arizona presented with 6 weeks of gradually worsening left-sided neck pain and stiffness accompanied 
by fevers, emesis, and limited cervical range of motion. He was otherwise healthy, with no previous medical history and an unremarkable birth history. He was exposed to dogs where he lived, and his immunizations were up to date. The patient had been treated at an outside facility 2 months earlier with 3 days of amoxicillin for presumed streptococcal pharyngitis. His symptoms improved, but he was readmitted to an outside hospital 2 weeks later with a presumed diagnosis of culture-negative meningitis, and subsequently treated with 10 days of amoxicillin. Four weeks later, his neck pain worsened, and he was started on prophylactic intravenous antibiotics after a lumbar puncture at an outside facility raised concern for possible bacterial meningitis. The CSF profile demonstrated a white blood cell count of 2160 cells $/ \mathrm{mm}^{3}$ (62\% neutrophils), a red blood cell count of 43 cells $/ \mathrm{mm}^{3}$, a glucose level of 30 $\mathrm{mg} / \mathrm{dl}$, a protein level of $252 \mathrm{mg} / \mathrm{dl}$, and a negative Gram stain and culture.

The patient was transferred to our institution, where CSF cultures were negative and intravenous antibiotics were discontinued. Magnetic resonance imaging of the brain and cervical spine revealed a Chiari Type I malformation with $17 \mathrm{~mm}$ of tonsillar descent, as well as an 11$\mathrm{mm}$ contrast-enhancing intradural extramedullary cervical mass, expanding the levels of $\mathrm{C}-1$ through $\mathrm{C}-3$. The lesion was eccentric to the left, causing $40 \%$ effacement of the spinal canal and moderate cord compression (Fig. $1 \mathrm{~A}$ and $\mathrm{B})$. On the basis of the imaging characteristics, the lesion was suspected to be intradural and neoplastic in nature. MRI results of the thoracic and lumbar spine were both normal. He was neurologically intact with full strength on admission, with a primary complaint of headache and left-sided neck pain.

\section{Operation}

The patient underwent a suboccipital craniectomy and laminectomies at C-1 through C-3 to decompress the Chiari malformation, perform a biopsy, and resect the enhancing lesion. The dura mater was opened over the cerebellar hemispheres in the typical "Y-shaped" fashion and extended inferiorly to the level of C-3 for intradural exploration. Subarachnoid dissection was performed between the cerebellar tonsils and brisk CSF outflow was visualized from the obex. Intradural exploration did not yield any evidence of tumor, but a large compressive mass was identified in the epidural space, extending laterally into the left C-2 and C-3 neural foramina. The well-encapsulated mass was incised and a significant amount of inflammatory tissue was immediately visualized, along with long yellow strands and thick yellow fluid. Multiple biopsy specimens were sent for pathological examination and the lesion was thoroughly debulked internally.

\section{Pathological Findings}

The lesion was recognized as a parasitic infection on intraoperative frozen section and reported to the Centers for Disease Control and Prevention. The parasite was identified as O. lupi (Fig. 2).

\section{Postoperative Course}

The patient remained neurologically intact postopera-
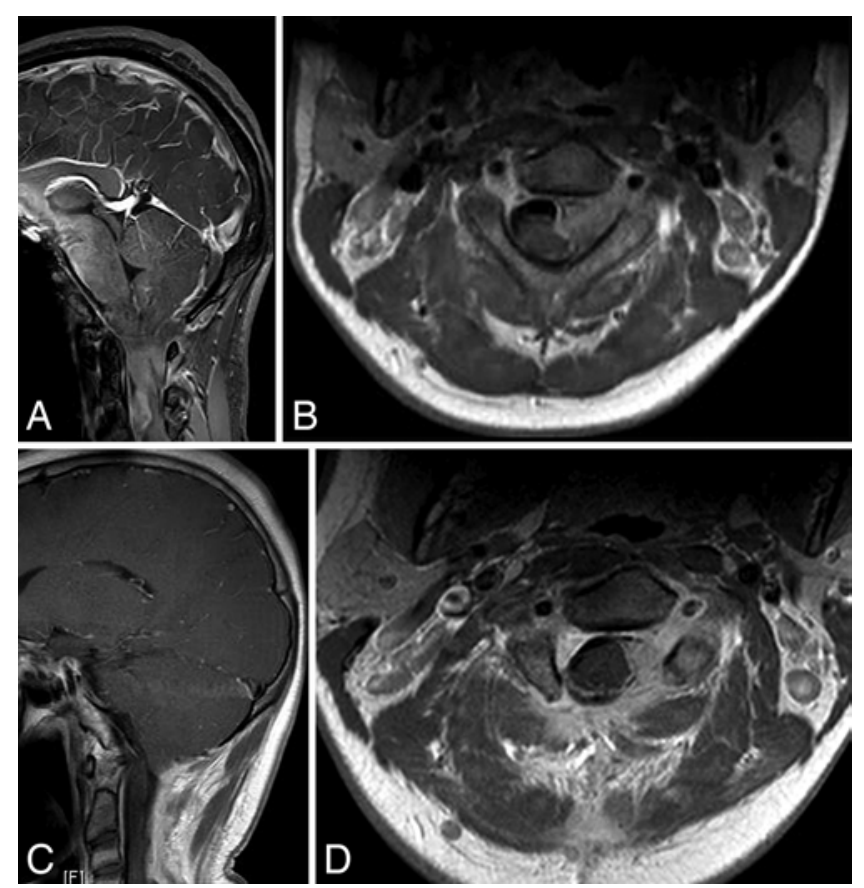

FIG. 1. Preoperative sagittal (A) and axial (B) MR images with $\mathrm{Gd}$ enhancement of the cervical spine show an 11-mm contrast-enhancing intradural extramedullary cervical mass, expanding the levels of C-1 to C-3. Postoperative sagittal (C) and axial (D) MR images at 6 weeks demonstrate a significant reduction in the size of the lesion and reduced mass effect on the spinal cord.

tively and was maintained on 2 weeks of dexamethasone to decrease the potentially negative effects of inflammatory products released from the lesion. Once the parasite was identified as $O$. lupi, the patient was started on 100 $\mathrm{mg}$ of oral doxycycline twice a day as recommended by the infectious diseases team, for a duration of 6 weeks. At the 1-month follow-up examination, the patient was doing well, with resolution of his preoperative symptoms, and at 6-week follow-up, MRI demonstrated that the enhancing lesion had decreased significantly in size (Fig. 1C and D).

Ten weeks after surgery, the patient returned to the emergency room with upper-extremity paresthesias and worsening neck pain. He had completed 6 weeks of doxycycline therapy. MRI of the cervical spine with contrast administration demonstrated a recurrent enhancing mass at the resection site with spinal cord compression (Fig. 3A and B). Given the size of the lesion and severity of spinal cord compression, the patient was taken to the operating room for reexploration and resection of this lesion. The initial resection cavity had expanded with purulent material and inflammatory tissue, causing significant tension and pressure on the adjacent dura. Long thick yellow strands were again visualized, and a biopsy specimen was confirmed as $O$. lupi infection again. The lesion was thoroughly debulked and the dura was not violated. The thecal sac was no longer under pressure after the decompression. The patient remained neurologically intact after surgery. At the 6-week follow-up after the second operation (4 months from the initial procedure), the patient was doing well and was neurologically intact, with resolution of his headaches and neck pain. He has since completed 


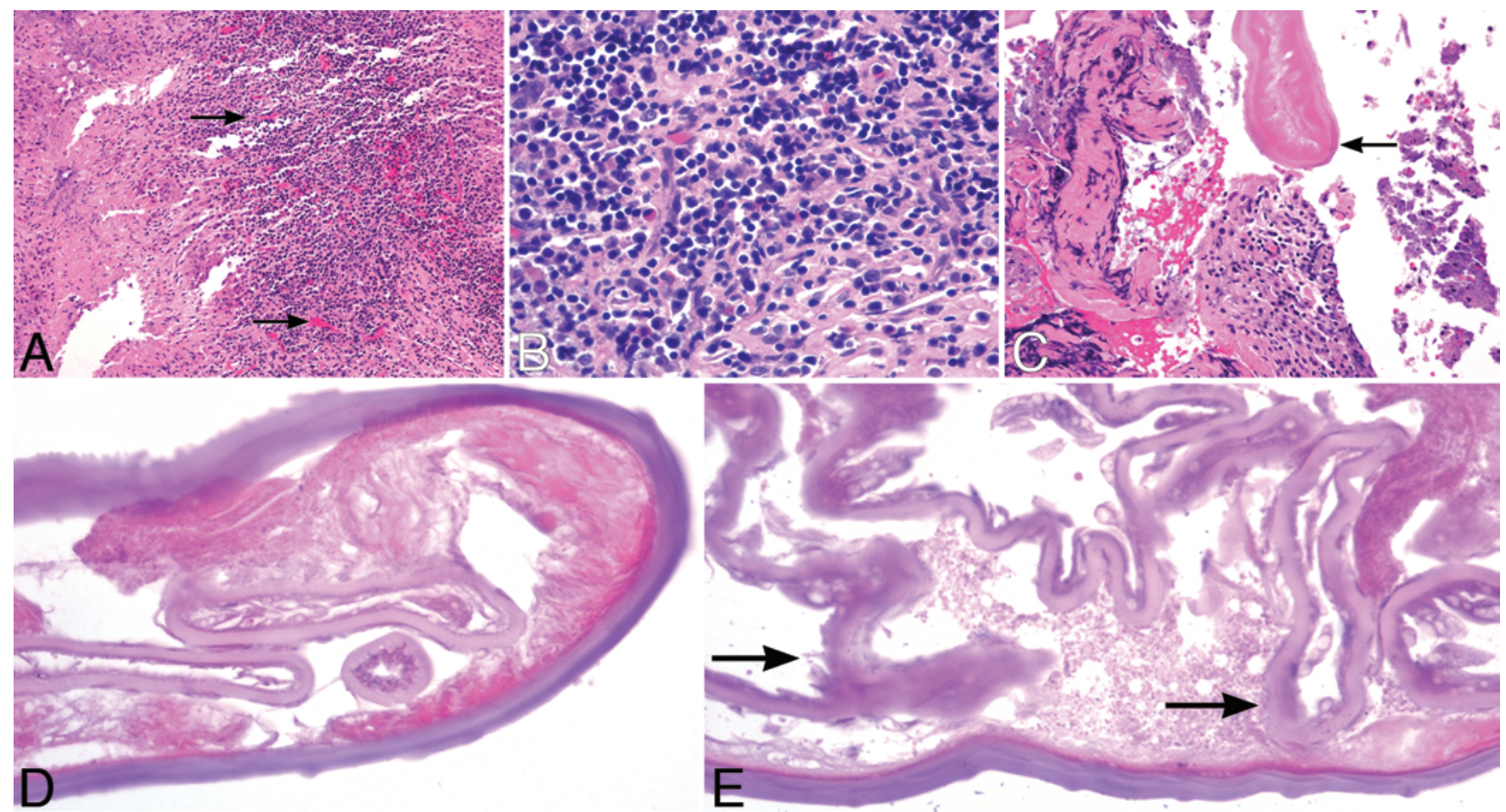

FIG. 2. Photomicrographs of the biopsy sections. A and B: Stained sections of the cyst capsule. Central necrosis surrounded by an inflammatory infiltrate of mononuclear cells and multinucleated giant cells is visible (A, arrows). A high-power view of these cells is also shown (B). C: A section of the nematode (arrow) adjacent to the inflammatory tissue is indicated. D and E: A cross-section of the nematode $(D)$ and a high-power view of the nematode's cuticular ridges ( $E$, arrows) are demonstrated. $H$ \& $E$. Original magnifications $\times 100(\mathrm{~A}), \times 400(\mathrm{~B}), \times 200(\mathrm{C})$, and $\times 400(\mathrm{D}$ and $\mathrm{E})$.

an additional 6 weeks of doxycycline therapy and has now transitioned to oral ivermectin $(9 \mathrm{mg})$ every 3 months. His most recent MRI session, 6 weeks after the second procedure, demonstrated a significant decrease in the size of the infectious lesion and decreased spinal cord compression (Fig. 3C and D).

\section{Discussion}

This is the second reported human case of $O$. lupi infection in the epidural cervical spine. The $O$. lupi species was first identified from a wolf in Russia (Canis lupus) in 1967. Since then, this strain has been known to infect dogs and other canines, with a primary presentation of either acute or chronic ocular infection. Cases of canine ocular onchocercosis have been increasingly reported in Europe and the US over the last several decades. Black flies and midges are suspected to be the vectors of transmission, and presumably may transmit the nematode to humans as well. Other Onchocerca infections identified include $O$. gutturosa (from cattle), O. jakutensis (deer in Austria), O. cervicalis (horses), and $O$. dewittei japonica (Japanese wild boar). Only $O$. cervicalis and $O$. gutturosa have presented in the ocular location in humans. All others have presented in subcutaneous tissues involving the knee, wrists, feet, head, neck, or abdomen. No other $\mathrm{On}$ chocerca species have been reported to infect the intradural or extradural space of the cervical spine. ${ }^{4-6,9,15,16,21,23}$

No prior reports have documented the treatment of CNS O. lupi infections in humans; however, ivermectin
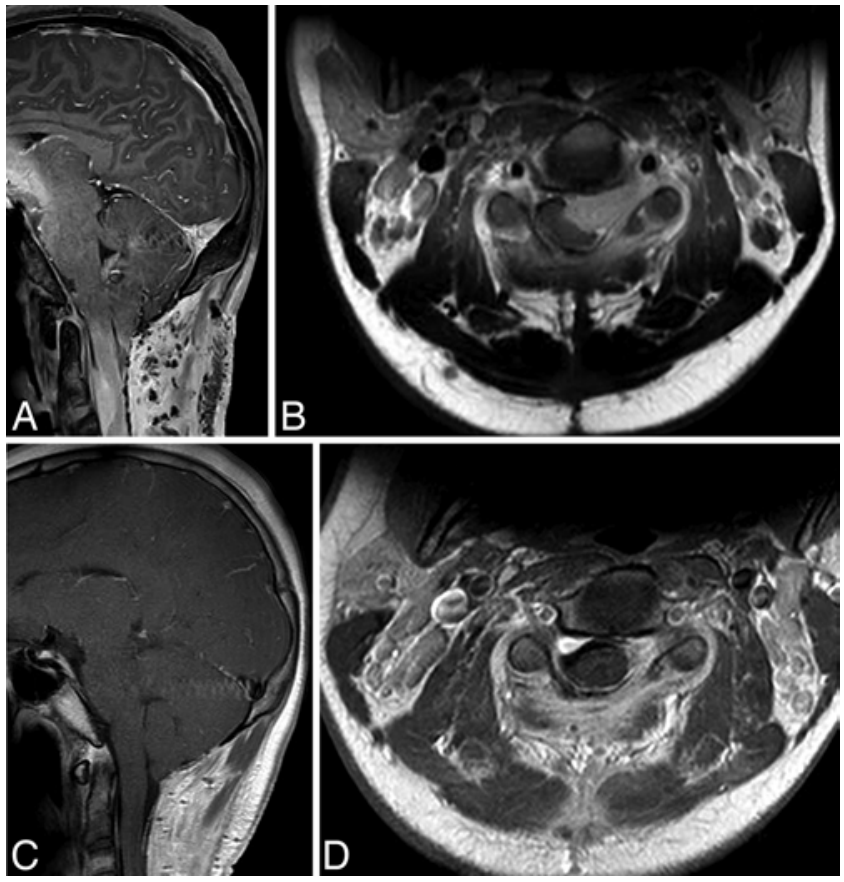

FIG. 3. Preoperative sagittal (A) and axial (B) MR images with Gd enhancement of the cervical spine show a recurrence of the enhancing extradural cervical mass with significant spinal cord compression. Postoperative sagittal (C) and axial (D) MR images at 6 weeks after the second operation demonstrate a significant reduction in the size of the lesion and reduced mass effect on the spinal cord. 
has been demonstrated to kill $O$. volvulus larvae and promote the death of adult worms when administered 4 times per year. The first patient diagnosed with $O$. lupi infection of the epidural cervical spine was also treated with ivermectin, ${ }^{5}$ and interestingly, was also a Native American residing in northeastern Arizona. She was a 22-month-old child presenting with progressive neck pain and stiffness and without neurological deficit. She was found to have an extradural lesion at C-2 to C-4 that was biopsied through a left-sided C2-4 laminectomy. She was initially treated with oral doxycycline and was subsequently transitioned to intravenous ivermectin injections. Her symptoms improved after surgery and eventually resolved by her 7-month follow-up examination at the time the case was reported. ${ }^{5}$ At her most recent 28-month follow-up with Dr. Feiz-Erfan, she was neurologically stable and remains on a regimen of ivermectin therapy 4 times per year.

Both reports describe patients from the pediatric population who initially presented with symptoms of meningitis and were unsuccessfully treated with amoxicillin. Therefore, in patients with refractory symptoms in which meningitis has been ruled out, MRI of the cervical spine with Gd should be obtained to rule out an infectious lesion. Infection must remain on the list of differential diagnoses for patients with a circumscribed enhancing spinal lesion and fever, and we recommend that these patients undergo immediate biopsy and attempted lesional resection to obtain a diagnosis and initiate appropriate treatment. If the lesion resides in the cervical epidural space without vertebral body invasion, this can be completed through a traditional posterior cervical laminectomy approach at the appropriate levels. If there is concern that an intradural component is present, the dura should be opened and explored. In this case there was a strong suspicion that the lesion was intradural, given no evidence of the capsule epidurally on initial inspection and exploration. When no lesion was found extradurally, release of CSF from the intrathecal space allowed decompression of the thecal sac and improved visualization in the lateral epidural space. This was a unique case, in that the patient also had a severe Chiari malformation, and the decision was made to open the dura for a Chiari decompression in conjunction with resection of the lesion. Thus, when the lesion was not initially visualized in the epidural space, the dural opening was extended inferiorly from the point of tonsillar decompression. If there had been no intention to open the dura to perform a Chiari decompression, more bone could have been removed laterally, allowing for further visualization of the lateral epidural space and potential visualization of the lesion. If we encounter this situation in the future in a patient without a concurrent Chiari malformation or other pathology necessitating a dural opening, we would avoid opening the dura to limit CSF exposure to the infection.

Once a diagnosis is made, and if a parasite is found and identified, targeted antiparasitic therapy should be initiated immediately. Corticosteroids should be administered postoperatively to reduce the negative effects of inflammatory products released from the cyst capsule. When possible, efforts should be made to keep the lesion capsule intact to limit the spread of inflammatory material. ${ }^{8,13,14}$ Our case demonstrates the importance of strict adherence to the postoperative antiparasitic therapy. Antiparasitic therapy with doxycycline alone in our patient was insufficient to treat and control the infection. The addition of ivermectin has adequately controlled the infection in the first patient identified with cervical onchocercosis, and thus far our patient has remained asymptomatic since initiating ivermectin therapy.

Given the common geographic setting of both reports, there may be an epidemiological association between the transmission of this parasite and the southwestern US. More case reports will be needed to define a true epidemiological risk. Due to a lack of standardized treatment regimens for cervical $O$. lupi infections, long-term clinical and radiological follow-up will be needed to monitor for continued progress and disease resolution in these patients.

\section{Acknowledgment}

We thank Steve Taylor, MHS, PA(ASCP), for providing the photomicrography.

\section{References}

1. Awasthy N, Chand K: Primary hydatid disease of the spine: an unusual case. Br J Neurosurg 19:425-427, 2005

2. Basáñez MG, Pion SD, Churcher TS, Breitling LP, Little MP, Boussinesq M: River blindness: a success story under threat? PLoS Med 3:e371, 2006

3. Berngard SC, Miller M: Salmonella spinal infection: a rare case in a patient with advanced AIDS. J Int Assoc Provid AIDS Care 12:241-244, 2013

4. Burr WE Jr, Brown MF, Eberhard ML: Zoonotic Onchocerca (Nematoda:Filarioidea) in the cornea of a Colorado resident. Ophthalmology 105:1494-1497, 1998

5. Eberhard ML, Ostovar GA, Chundu K, Hobohm D, FeizErfan I, Mathison BA, et al: Zoonotic Onchocerca lupi infection in a 22-month-old child in Arizona: first report in the United States and a review of the literature. Am J Trop Med Hyg 88:601-605, 2013

6. Egyed Z, Sréter T, Széll Z, Beszteri B, Oravecz O, Márialigeti K, et al: Morphologic and genetic characterization of Onchocerca lupi infecting dogs. Vet Parasitol 102:309-319, 2001

7. Fkih L, Boussoffara L, Bedoui SA, Saad S, Belhabib D, Hassène $\mathrm{H}$, et al: [Echinococcosis of the rib with epidural extension.] Rev Pneumol Clin 65:169-172, 2009 (Fr)

8. Iacoangeli M, Moriconi E, Gladi M, Scerrati M: Isolated cysticercosis of the cauda equina. J Neurosci Rural Pract 4 (Suppl 1):S117-S119, 2013

9. Ilhan HD, Yaman A, Morishima Y, Sugiyama H, Muto M, Yamasaki H, et al: Onchocerca lupi infection in Turkey: a unique case of a rare human parasite. Acta Parasitol 58:384-388, 2013

10. Kafaji A, Al-Zain T, Lemcke J, Al-Zain F: Spinal manifestation of hydatid disease: a case series of 36 patients. World Neurosurg 80:620-626, 2013

11. Karadereler S, Orakdögen M, Kiliç K, Ozdogan C: Primary spinal extradural hydatid cyst in a child: case report and review of the literature. Eur Spine J 11:500-503, 2002

12. Kotil K, Tatar Z, Bilge T: Spinal hydatidosis accompanied by a secondary infection. Case report. J Neurosurg Spine 6:585-590, 2007

13. Neumayr A, Tamarozzi F, Goblirsch S, Blum J, Brunetti E: Spinal cystic echinococcosis - a systematic analysis and review of the literature: part 1. Epidemiology and anatomy. PLoS Negl Trop Dis 7:e2450, 2013

14. Neumayr A, Tamarozzi F, Goblirsch S, Blum J, Brunetti 
E: Spinal cystic echinococcosis - a systematic analysis and review of the literature: part 2. Treatment, follow-up and outcome. PLoS Negl Trop Dis 7:e2458, 2013

15. Orihel TC, Ash LR, Holshuh HJ, Santenelli S: Onchocerciasis in a California dog. Am J Trop Med Hyg 44:513-517, 1991

16. Otranto D, Sakru N, Testini G, Gürlü VP, Yakar K, Lia RP, et al: Case report: First evidence of human zoonotic infection by Onchocerca lupi (Spirurida, Onchocercidae). Am J Trop Med Hyg 84:55-58, 2011

17. Salduz A, Koyuncu LO, Dikici F, Talu U: [Long-term result of treatment for paraspinal and extradural hydatid cyst: a case report.] Acta Orthop Traumatol Turc 43:267-271, 2009 (Turkish)

18. Sapkas GS, Machinis TG, Chloros GD, Fountas KN, Themistocleous GS, Vrettakos G: Spinal hydatid disease, a rare but existent pathological entity: case report and review of the literature. South Med J 99:178-183, 2006

19. Senol MG, Tekeli H, Kendirli MT, Kaya S, Turhan V, Sonmez G, et al: Intramedullary hydatid cyst of the cervical spine. Indian J Med Microbiol 30:480-481, 2012

20. Sharma NK, Chitkara N, Bakshi N, Gupta P: Primary spinal extradural hydatid cyst. Neurol India 51:89-90, 2003

21. Sréter T, Széll Z: Onchocercosis: a newly recognized disease in dogs. Vet Parasitol 151:1-13, 2008
22. Yoo M, Lee CH, Kim KJ, Kim HJ: A case of intraduralextramedullary form of primary spinal cysticercosis misdiagnosed as an arachnoid cyst. J Korean Neurosurg Soc 55:226-229, 2014

23. Zarfoss MK, Dubielzig RR, Eberhard ML, Schmidt KS: Canine ocular onchocerciasis in the United States: two new cases and a review of the literature. Vet Ophthalmol 8:5157, 2005

\section{Author Contributions}

Conception and design: Bhardwaj, Chen, Theodore. Acquisition of data: Chen. Analysis and interpretation of data: Chen, Moon, deMello. Drafting the article: Chen. Critically revising the article: Bhardwaj, Moon, deMello, Feiz-Erfan, Theodore. Reviewed submitted version of manuscript: Bhardwaj. Study supervision: Bhardwaj, Theodore.

\section{Correspondence}

Ratan D. Bhardwaj, c/o Neuroscience Publications, Barrow Neurological Institute, St. Joseph's Hospital and Medical Center, 350 W. Thomas Rd., Phoenix, AZ 85013. email: neuropub@dignity health.org. 